rev.relac.int.estrateg.segur.6(2):21-34,2011

\title{
EVOLUCIÓN DEL RÉGIMEN DE CONTROL Y FINANCIACIÓN DEL TERRORISMO*
}

\author{
Fabio Sánchez Cabarcas**
}

\section{RESUMEN}

Este artículo analiza la evolución del régimen contra la financiación del terrorismo en el sistema internacional. Esta actividad ha sido compleja de definir y controlar, no obstante, durante el siglo XX, diversos actores del sistema internacional, bajo el liderazgo de la ONU han desarrollado mecanismos institucionales y transnacionales para responder y limitar su alcance. En el transcurso del trabajo se evidencia la consolidación de un régimen que lucha frente a un fenómeno que cuenta con innumerables recursos para mimetizarse en la era tecnológica y que tiene la capacidad de afectar el orden mundial.

Palabras clave: Régimen internacional, terrorismo, neoinstitucionalismo, ONU.

* Este trabajo es producto de la investigación: Evolución del terrorismo en el sistema internacional, de la línea: "Seguridad Humana y Agenda Global" adscrita al Grupo de Análisis Político-GAP- de la Escuela de Política y Relaciones Internacionales de la Universidad Sergio Arboleda.

** Doctorando, Máster y Diploma en Estudios Avanzados (DEA) en Relaciones Internacionales con Suficiencia Investigadora de la Universidad Autónoma de Barcelona. Comunicador Social-Periodista y Magíster en Relaciones Internacionales de la Pontificia Universidad Javeriana. National Security Institute, NSI-2010, Universidad de Delaware. Coordinador del Área de Relaciones Internacionales y Profesor-Investigador de la Escuela de Política y Relaciones Internacionales de la Universidad Sergio Arboleda, Bogotá.fabio.sanchez@usa.edu.co 


\begin{abstract}
This article analyzes the evolution of a regime against terrorist financing in the international system. This activity has been difficult to define and control; however, during the twentieth century, various actors in the international system under the leadership of the UN, have developed transnational and institutional mechanisms to respond and limit its scope. The present study shows the consolidation of a regime that struggles with an adversary that has countless technological resources at hand and has the ability to affect world order.
\end{abstract}

Key Words: International regimes, terrorism, neoinstitutionalism, UN.

\title{
RESUMO
}

Este artigo analisa a evolução do regime contra o financiamento do terrorismo no sistema internacional. Esta atividade tem sido muito difícil de definir e controlar, no entanto, durante o século XX, vários atores do sistema internacional, sob o comando da ONU, desenvolveram mecanismos institucionais e transnacionais para responder e limitar seu alcance. No decorrer do trabalho, torna-se evidente a consolidação de um regime que luta diante de um fenômeno que conta com inumeráveis recursos para mimetizar-se na era tecnológica e que tem a capacidade de afetar a ordem mundial.

Palavras chave: Regime internacional, terrorismo, neoinstitucionalismo, ONU.

\section{INTRODUCCIÓN}

La literatura sobre regímenes es amplia en la disciplina de las Relaciones Internacionales (Jervis, 1982; Keohane, 1982; Krasner, 1983; Haggard \& Simmons, 1987; Buzan, 1993; Hasenclever, et al., 1997). Su estudio se ha desarrollado de forma sistemática desde la década de 1980. En aquel entonces, Keohane era enfático en su importancia: "Estudiamos regímenes porque estamos interesados en comprender el orden de la política mundial" (Keohane, 1982, p. 325). La definición más precisa, y en la que coinciden diferentes autores, es: "Normas, reglas, principios y procesos de toma de decisiones, alrededor de los cuales convergen las expectativas de los actores en un área determinada de Relaciones Internacionales" (Krasner, 1983, p. 2).

A partir de la anterior definición, este artículo analiza la evolución del régimen de control y la financiación del terrorismo, y sus cambios en fuerza, forma organizacional, alcance y dotación, aspectos vitales en este tipo de estudios (Haggard \& Simmons, 1987). En la primera parte, se analiza el origen del terrorismo como problema del sistema internacional, sus primeras manifes- 
taciones y los primeros intentos por controlarlo. En la siguiente sección se identifican las diversas y complejas modalidades que utilizan ciertos actores del sistema internacional para financiar actos terroristas, y los mecanismos de control que se han establecido para combatirlas. En la tercera parte, se destaca el papel preponderante de la ONU en la consolidación del régimen en el transcurso del siglo XX. Posteriormente, se analiza cómo el régimen evoluciona hasta conseguir sanciones a grupos y personas específicas. Finalmente, las conclusiones.

\section{ORIGEN Y DEFINICIÓN DEL TERRORISMO COMO PROBLEMA INTERNACIONAL CONTEMPORÁNEO}

Los ataques del 11-S en Nueva York evidenciaron la enorme capacidad de daño que pueden ocasionar las redes terroristas modernas. Sus alcances superan de forma dramática los atentados que décadas atrás habían cometido diversas organizaciones terroristas a través del mundo. Desde aquél día, la sensación de inseguridad ha crecido y todo indica que gran parte de la población mundial está bajo un estado psicológico de miedo e incertidumbre gracias a lo que algunos teóricos catalogan como terrorismo global (Mowlan, 2007, p. 1). Controlar su financiación ha sido un reto muy grande, incluso antes del 11-S, varias medidas multilaterales dentro de la $\mathrm{ONU}$, y otras como el Grupo de Acción Financiera (GAFI) ${ }^{1}$, explican la preocupación que este tema generaba en la agenda mundial durante la Guerra Fría. A continuación, se presentan algunos antecedentes históricos del problema.

El terrorismo no es un fenómeno nuevo, a través de archivos históricos se pueden encontrar documentos que muestran cómo las conductas de los zelotes sicarios de la Judea romana, los asesinos ismaelitas de la Edad Media, o los thugs de la India colonial, asesinaban con sorpresa y generaban miedo en la población. No obstante, el término terrorismo se utilizó por primera vez en 1795 para hacer referencia al régimen instaurado por los jacobinos en la Francia postrevolucionaria (Jordan, 2004, p. 13). Luego, en siglo XIX, se utilizó para definir la violencia de los irlandeses en contra de los ingleses, y para señalar al grupo ruso Narodnaya Volya (voluntad del pueblo), que en 1881 asesinó al zar Alejandro II (Marsella, 2004, p. 20).

Pero, ien qué momento se le ve como un problema de la sociedad moderna? Luego de la muerte en Marsella del Rey Alejandro I de Yugoslavia y de Louis Barthou, Ministro de Relaciones Exteriores francés, el país galo en 1934 organizó por primera vez una convención internacional para la supresión del terrorismo, que tres años más tarde (1937), concibió la firma de dos tratados: i) la Convención para la prevención y represión del terrorismo y, ii) la Convención para la creación de un Tribunal Penal Internacional. Acciones que tuvieron poco éxito, bajo el mando

1. Financial Action Task Force (FATF, por sus siglas en ingles). Para más información véase su sitio en internet: www.fatfgafi.org/ 
de la poco eficiente Liga de Naciones, que no logró detener el estallido de la Segunda Guerra Mundial (Hinojosa, 2008, p. 13).

Durante la Guerra Fría, en la década de 1970, las Naciones Unidas empezaron a preocuparse por combatir el terrorismo internacional. Con la Resolución 2625 de ese mismo año, la Asamblea General (AG) recomendó a todo estado: "abstenerse de organizar, instigar, ayudar o participar (...) en actos de terrorismo en otros estados, o de consentir actividades organizadas dentro de su territorio, encaminadas a cometer dichos actos" (Hinojosa, 2008, p. 15). De la misma manera, la (AG) a través de la Resolución $2645^{2}$ contempla la desviación a mano armada de aeronaves, o injerencia en los viajes aéreos civiles, debido a los casos de secuestro de aviones que se presentaban en aquella época, por algunas organizaciones terroristas en Oriente Medio, una de estas bajo el mando del posteriormente reconocido líder palestino Yasser Arafat, y otras bajo órdenes de terroristas libios. Luego, en 1972 la (AG) organizó el primer Comité Especial sobre el "terrorismo internacional", que tuvo como resultado la Resolución 3034, la cual ya incluía más elementos de lucha en contra de ese fenómeno, y dicho Comité presentaría tres informes en 1973, 1977 y $1979^{3}$.

En esta misma década, en Europa se empezó a revisar con mayor atención el problema del terrorismo. Más aún, luego del secuestro del equipo israelí en las Olimpiadas de Munich el 5 de septiembre de 1972, que dejó como saldo 17 personas muertas: 11 atletas israelíes, un policía alemán y cinco terroristas (Reeve, 2000). Este hecho alarmó a la opinión pública mundial, sobre los alcances que podría tener el terrorismo internacional y generó un proceso que se inició en 1975 con el Consejo de Ministros del Interior de los 9 países que en aquel entonces conformaban la Comunidad Económica Europea (CEE), quienes mantuvieron conversaciones e intercambiaron información sobre la problemática terrorista, delincuencia organizada, orden público y materiales explosivos, hasta que el 29 de junio de 1976 se configuraron en Luxemburgo los Grupos Trevi, los cuales hicieron la cooperación en estos temas permanente (Bunyan, 1993)4.

A continuación, se verá la evolución del régimen de control de financiación del terrorismo, revisando los hechos que han marcado su desarrollo a través de la historia y analizando cómo el

2. Res. 2645 (XXV), (AG) ONU Doc. A/8028 (1970).

3. "Medidas para prevenir el terrorismo internacional que pone en peligro vidas humanas inocentes o causa su pérdida, o compromete las libertades fundamentales, y estudio de las causas subyacentes de las formas de terrorismo y los actos de violencia que tienen su origen en las aflicciones, la frustración, los agravios, y la desesperanza y que conducen a algunas personas a sacrificar vidas humanas, incluida la propia, en un intento de lograr cambios radicales", (AG) Res. 3034 (XXVII), ONU AGDO, 27 mo per. de sesiones, 2114ta sesión, Sup. No. 30, en 19, ONU Doc. A/8730 (1972). Sobre los tres informes: 1973 (ONU AGDO, 28vo per. de sesiones, Sup. No. 28, ONU Doc. A/9028); 1977 (ONU AGDO, 32do per. de sesiones, Sup. No. 37, ONU Doc. A/32/37) y 1979 (ONU AGDO 34to per. de sesiones, Sup. No. 37, ONU Doc. A/34/37).

4. En aquél momento eran motivo de preocupación las acciones terroristas de ETA en España, las Brigadas Rojas en Italia y el IRA en Gran Bretaña. 
11-S fue un catalizador que centra el interés de los actores y la opinión pública internacional sobre la problemática (Byers, 2002).

\section{2. ¿CÓMO EVOLUCIONA LA FINANCIACIÓN DEL TERRORISMO TRANSNACIONAL Y EL RÉGIMEN PARA CONTROLARLO, A FINALES DEL SIGLO XX, Y PRINCIPIOS DEL XXI?}

Debido a la globalización de los mercados financieros internacionales, los grupos terroristas utilizan diversas modalidades para financiarse y ocultar el lucro procedente de diversas actividades delictivas. Algunas de estas organizaciones funcionan como multinacionales, con una logística específica y compuesta por personas, armas e itinerarios definidos.

De la misma manera, estos grupos pueden controlar desde el tráfico de armas y drogas hasta la trata de personas. Hacen uso de los servicios de internet y otras tecnologías como el GPS para localizar envíos (de armas o dinero), en zonas alejadas o para concretar reuniones en "estados fallidos" o parajes inhóspitos del primer mundo, algunas de estas acciones son catalogadas como ciberdelitos. Estos mecanismos facilitan la práctica de un "crimen transnacional" con capacidad para permear las fronteras convencionales, y ejecutar atentados de gran espectacularidad como los ocurridos en Nueva York (2001), y Madrid (2004), que contrario a lo que se pudiera pensar no fueron muy costosos ${ }^{5}$.

Se debe tener en cuenta que algunos grupos más pequeños se financian de forma privada, y es aquí en donde se presenta uno de los principales obstáculos para controlar el problema: determinar y detectar cuándo las actividades que generan dinero son lícitas o ilícitas. Por ejemplo, algunas organizaciones terroristas cuentan con un sistema empresarial que les da dinero, empleo a algunos de sus miembros y cubrimiento a sus movimientos comerciales, tal y como se sospecha de ciertas empresas que en el País Vasco cooperan con ETA (Hinojosa, 2008, p. 79) ${ }^{6}$, lo mismo que ocurría con las inversiones de la familia Bin Laden en Arabia Saudita y Sudán hace algunos años. Otra modalidad lícita, un poco más particular, la constituyen los aportes de rifas y donaciones a entidades sin ánimo de lucro, que luego destinan parte del dinero a los grupos al margen de la ley, caso también de los aportes a institutos como el Centro Cultural Islámico en Madrid que recibía antes del 11-M, importantes donaciones de saudíes desconocidos (Hinojosa, 2008, p. 91).

5. Según datos de un seminario sobre financiación del terrorismo, celebrado en el Instituto Universitario Ortega y Gasset (14 de noviembre de 2005) el costo del 11-S en Nueva York fue de 500 mil dólares y el del 11-M en Madrid, 106.000 euros aproximadamente, para más información véase: (Hinojosa, 2008, p. 92).

6. Este autor profundiza más al respecto, sobre cómo ETA instaba a la comunidad para la compra de productos "autóctonos", es decir, de empresas que les aportaban dinero, y por otra parte, sobre cómo la presencia de Batasuna en la Cámara Legislativa Vasca, ayuntamientos y Juntas Generales de las Diputaciones, les permitió acceso directo a recursos públicos. 
De acuerdo a lo anterior, resulta pertinente la clasificación a la que hace referencia Curia:

Tabla 1. Esquemas Financiación del Terrorismo Internacional

\begin{tabular}{|l|l|}
\hline People support & Población local financia algún movimiento \\
\hline State support & Estado que financia (difícil comprobación) \\
\hline Crime support & Narcotráfico, secuestro, extorsión \\
\hline Clean support & $\begin{array}{l}\text { Mantenimiento de hospitales, casas de huérfanos, } \\
\text { centros culturales de adoctrinamiento. }\end{array}$ \\
\hline
\end{tabular}

Fuente: (Curia, 2005) Elaboración propia.

Por otra parte, desde finales de la década de 1980, la comunidad internacional ha estado luchando en contra de la, tal vez más común, forma ilícita de financiación de redes terroristas: el blanqueo de dinero. Una de las principales respuestas a este problema ha sido el $\mathrm{GAFl}^{7}$, creado en la Cumbre de los Siete (G-7) en 1989 en París, y el cual en 1990 expidió un conjunto de 40 recomendaciones $^{8}$, a las que luego del 11 -S se le sumarían 9 más de carácter especial, acordadas en Washington el 30 de octubre de 2001, estas son:

1. Ratificación y ejecución de los instrumentos de la ONU

2. Tipificación de la financiación del terrorismo y el blanqueo asociado

3. Congelamiento y decomiso de activos terroristas

4. Informe de transacciones sospechosas relativas al terrorismo

5. Cooperación internacional

6. Sistemas alternativos de envíos de fondos

7. El GAFI cuenta con 33 miembros, 31 países 2 instituciones internacionales (Consejo de Cooperación del Golfo (GCC) y la Comisión Europea. En su web site se define así: "The Financial Action Task Force (FATF) is an inter-governmental body whose purpose is the development and promotion of national and international policies to combat money laundering and terrorist financing. The FATF is therefore a "policy-making body" created in 1989 that works to generate the necessary political will to bring about legislative and regulatory reforms in these areas. The FATF has published $40+9$ Recommendations in order to meet this objective". Para más información véase: www.fatf-gafi.org

8. Después de ser publicadas, las recomendaciones del GAFI fueron completamente revisadas en 1996 y luego en 2003. Éstas instan a los estados a: i) implementar las convenciones relevantes a su respectiva legislación, ii) condenar el lavado de dinero y brindar herramientas legales a las autoridades para la confiscación de los ingresos producidos por el lavado de dinero, iii) implementar políticas para los usuarios de los bancos (verificación de la identidad personal), iv) guardar un registro de las transacciones sospechosas de instituciones financieras, de negocios y no financieras, v) establecer una unidad de inteligencia financiera, que reciba y procese los reportes de transacciones sospechas o dudosas y, vi) cooperar con la comunidad internacional investigando y facilitando la prosecución del lavado de dinero. 
7. Transferencias por cable

8. Organizaciones sin fines de lucro

9. Envío de dinero por personas (cross border)

El GAFI es un ejemplo evidente de multilateralismo en el sistema internacional contemporáneo. En 1998 tras ocho años de vida, decidió abrirse y comprometer a algunas potencias emergentes: los BRIC's (Brasil, Rusia, India, China) junto con México y Sudáfrica. Así mismo, ha integrado con éxito dos organizaciones regionales: la Comisión Europea y el Consejo de Cooperación del Golfo (GCC, por sus siglas en inglés), compuesto por Bahrein, Kuwait, Omán, Qatar, Arabia Saudita y los Emiratos Árabes Unidos, además cuenta con observadores importantes: China, el Fondo Monetario Internacional y el Banco Mundial ${ }^{9}$.

Aunque en ocasiones no se logre comprobar la relación de los movimientos bancarios con los atentados, sí se logra capturar a los criminales que buscan "lavar" constantemente el dinero. Según Hinojosa, se pueden identificar tres fases del blanqueo de dinero:

a) La colocación: dinero en efectivo procedente de actividades delictivas es introducido en el circuito financiero.

b) El enmascaramiento (layering): transformación de los fondos a través de distintas operaciones que pretenden borrar el rastro del origen ilegal de dinero.

c) La integración: mediante la cual el dinero vuelve a los mercados financieros formales con una apariencia de legalidad.

Una vez finalizada la Guerra Fría en 1994, la ONU adoptó una Declaración sobre medidas para eliminar el terrorismo internacional ${ }^{10}$. Dos años después, en 1996, la Asamblea General estableció un nuevo comité especial cuyo propósito era elaborar convenios internacionales sobre terrorismo, del cual nació el Convenio Internacional para la Represión de la Financiación del Terrorismo.

9. Otros organismos multilaterales prestan ayuda, por ejemplo el grupo Egmont de Unidades de Inteligencia Financiera y, el Programa Global de Lavado de Dinero de la ONU. Otros organismos regionales integran también a otros estados menos poderosos, pero no por esto menos susceptibles de financiar terrorismo, por ejemplo en el Caribe el GAFIC (Grupo de Acción Financiera del Caribe); en América del Sur el GAFISUD (Grupo de Accion Financiera de Suramérica); en Europa Central el MONEYVAL (Committee of Experts on the Evaluation of Anti-Money Laundering Measures and the Financing of Terrorism) (en el marco del Consejo de Europa); y el APG (Asia Pacific Group), entre otros. De la misma manera, tanto el Fondo Monetario Internacional (FMI) como el Banco Mundial (BM), como parte de sus programas de mejoramiento, han apropiado las últimas recomendaciones del GAFI en cuanto a procedimientos, documentación y evaluaciones como una muestra de confianza.

10. Res. 49/60, (AG) (1994); y la Declaración complementaria de la Declaración de 1994 sobre medidas para eliminar el terrorismo internacional: Res. 51/210, (AG) Doc. A/Res/51/210 (1996). 
Es evidente que la financiación del terrorismo transnacional contemporáneo es un tema complejo de la actual agenda internacional. Se mimetiza con facilidad ante las medidas que han adoptado los estados, y gracias a los avances tecnológicos se hace cada día más sofisticada y difícil de contener. Veamos cómo se ha afrontado este reto en el marco de la ONU.

\section{EVOLUCIÓN DE LA LUCHA CONTRA LA FINANCIACIÓN DEL TERRORISMO EN LA ONU}

Luego de la segunda fase de la Guerra Fría, a principios de la década de 1980, se dan los primeros pasos en la ONU para detener el financiamiento de la delincuencia organizada. En aquél entonces, se limitaba a detener el blanqueo de dinero proveniente del tráfico de drogas. Prueba de esto es la Resolución 39/141 (AG) de 1984, con la que se buscó la elaboración de un proyecto de convenio contra el narcotráfico, proceso que culminó en 1988 en Viena, al adoptarse la Convención de Naciones Unidas contra el tráfico ilícito de estupefacientes y sustancias psicotrópicas (Hinojosa, 2008, p. 102).

A finales de la década de 1980, era evidente la fuerte conexión entre el blanqueo de dinero y el crimen transnacional organizado, ya no se trataba sólo del tráfico de estupefacientes. Por lo tanto, la Resolución 53/111 (AG) de 1999 creó un comité intergubernamental para que elaborara una convención internacional de carácter general contra la delincuencia internacional. El proyecto se consolidó con la Convención de Naciones Unidas contra la Delincuencia Organizada Transnacional, aprobada con la Resolución 55/25 (AG) del 8 de enero de 2001, la cual para marzo de 2007 contaba con la firma de 147 estados, y 132 eran parte de la misma (Hinojosa, 2008, p. 106), siendo el instrumento jurídico vinculante más efectivo en la ONU junto con la posterior Resolución 1373 del Consejo de Seguridad (CS) del 28 de septiembre de 2001.

Pero es el Convenio Internacional para la Represión de la Financiación del Terrorismo de 1999 el que aprueba mediante la Resolución 54/109 (AG), que se consiga por vez primera una definición de atentado terrorista:

(Art.2.1) (...) cualquier acto destinado a causar (...) lesiones corporales graves (...) a cualquier persona que no participe directamente en las hostilidades en una situación de conflicto armado, cuando el propósito de dicho acto (...) sea intimidar a una población u obligar a un gobierno o a una organización internacional a realizar un acto o a abstenerse de hacerlo" (Hinojosa, 2008, p. 112).

En la disciplina de las Relaciones Internacionales, la definición del fenómeno es más compleja aún ${ }^{11}$. 
En el mismo orden, el convenio determina que la financiación del terrorismo tiene que ver con la recolección de fondos, con la intención de sufragar actividades terroristas, y además considera delictiva cualquier tipo de complicidad relacionada con esa actividad, sin que sea necesario que los fondos "se hayan usado efectivamente" para llevar a cabo el acto delictivo (Hinojosa, 2008, p. 113), lo cual representa un avance significativo en la lucha contra este problema. De la misma manera, el Artículo 18 indica que los estados parte deben adoptar dos tipos de medidas preventivas que eviten la captación de dinero para actos terroristas en su territorio: i) prohibir actividades de personas y empresas que promuevan u organicen actividades terroristas $y$, ii) exigir a las entidades financieras la identificación de sus clientes y la transmisión de información sobre cualquier transacción sospechosa. El convenio resalta que para cumplir estos objetivos es adecuado seguir las recomendaciones del GAFI contra el blanqueo de dinero, entre las que se pueden destacar:

a) identificación de los clientes de entidades financieras

b) obligación de guardar los registros de operaciones durante cinco años

c) obligación de informar a las autoridades

\section{Las sanciones financieras a grupos y personas específicas}

La década de 1990 ofrece un panorama de constantes desafíos a la ONU. Los desequilibrios propios de la posguerra fría en países de la periferia, incluso en Europa (separación de la ex Yugoslavia), le exigió medidas oportunas y puntuales. Antes del fin de la Guerra Fría, el Consejo de Seguridad sólo había impuesto sanciones en dos oportunidades: contra Rhodesia del Sur (1966-1979) y contra Sudáfrica (1977-1994).

Las primeras sanciones de esta era fueron para el Irak de Hussein que invadió a Kuwait, y que posteriormente fue derrotado fácilmente. No obstante, el régimen brutal del dictador continuó durante varios años más; pese a la crisis en salud, necesidades básicas y calidad de vida del pueblo iraquí que generaron una aguda situación humanitaria, razón por la cual estas sanciones de la Resolución 661/1990 (CS) se conocen como SMD (Sanctions of Mass Destruction), a pesar de haber sido atenuadas por el "Programa Petróleo por Alimentos" aprobado mediante la Resolución

11. Keohane manifiesta que a pesar de que el Consejo de Seguridad de la ONU ha expedido resoluciones en contra del terrorismo, no ha tenido la capacidad de definirlo. Para él la definición más apropiada hasta el momento es la de Alex Schmid: "an anxiety-inspiring method of repeated violent action, employed by (semi)clandestine individual, group or state actors, for idiosyncratic, criminal, or political reasons, whereby-in contrast to assassination-the direct targets of violence are not the main targets" (Schmid, 1993, citado en; Keohane, 2002, p. 30). Por su parte Wight, quien también reconoce la dificultad, sugiere como mínimo cuatro aspectos para ser tenidos en cuenta si se busca una definición: i) es una forma de comunicación política violenta, ii) es siempre violencia ilegítima, iii) se trata de ataques deliberados contra instituciones y actores no-estatales y, iv) las víctimas no son los receptores del mensaje político (Wight, 2009, p. 102). Por su parte, Joseph aborda la problemática de su estudio desde una perspectiva crítica, diferenciando ontología y epistemología (Joseph, 2009). 
986/1995 (CS), el cual más adelante se vería manchado por un escándalo de corrupción en donde estaba implicado el hijo del Secretario General de la ONU de aquél entonces, Kofi Annan ${ }^{12}$.

Ante estos problemas, el Consejo de Seguridad decide delimitar y hacer más efectivas las sanciones contra actores no estatales. Es decir, individuos y núcleos familiares de dirigentes de estados corruptos o beligerantes, que tengan la capacidad de desestabilizar la paz y seguridad de alguna región del sistema internacional. Este tipo de sanciones han sido catalogadas como "inteligentes" (smart sanctions), porque buscan ante todo no afectar a la población civil. Se pueden destacar las siguientes:

\section{Tabla 2. Resoluciones del Consejo de Seguridad (Actividades Financieras)}

\begin{tabular}{|c|c|c|}
\hline Resolución & País & Medidas \\
\hline Res. 864/1993 (CS) & Angola & Contra UNITA. \\
\hline Res. 942/1994 (CS) & Bosnia & Contra los serbios de Bosnia. \\
\hline Res. 1127/1997 (CS) & Angola & $\begin{array}{l}\text { Lista de altos mandos y adultos de familias de de } \\
\text { UNITA. }\end{array}$ \\
\hline Res. 1132/1997 (CS) & Sierra Leona & $\begin{array}{l}\text { Congelación de Fondos en el ext. de Junta Mili- } \\
\text { tar de Sierra Leona y familiares. }\end{array}$ \\
\hline Res. 1173/1998 (CS) & Angola & $\begin{array}{l}\text { Congelación de Fondos en el ext. de UNITA y } \\
\text { familiares. }\end{array}$ \\
\hline Res. 1267/1999 (CS) & Afganistán & $\begin{array}{l}\text { Sanciones financieras }+ \text { bloque aéreo, al régi- } \\
\text { men Talibán. }\end{array}$ \\
\hline Res. $1333 / 2000$ & $\frac{\text { Sistema }}{\text { Internacional }}$ & $\begin{array}{l}\text { Congelación de fondos de Bin Laden + entida } \\
\text { des + socios + Al Qaeda. }\end{array}$ \\
\hline Res. 1343/2001 (CS) & Liberia & $\begin{array}{l}\text { Altos funcionarios del gobierno y cónyuges, mili- } \\
\text { tares y cónyuges. }\end{array}$ \\
\hline Res. 1483/2003 (CS) & Irak & $\begin{array}{l}\text { Saddam Hussein miembros de su familia y fun- } \\
\text { cionarios del régimen anterior. }\end{array}$ \\
\hline Res. 1532/2004 (CS) & Liberia & $\begin{array}{l}\text { Charles Taylor, determinados miembros de su } \\
\text { familia (nombre }+ \text { apellido). }\end{array}$ \\
\hline
\end{tabular}

Fuente: (Hinojosa, 2008, p. 120) Elaboración propia.

12. Sobre la relativa efectividad de sanciones véase: (Pape, 1997, 1998; Drezner, 1999; Mueller \& Mueller, 1999). Una posición a favor en (Lopez \& Cortright, 2004). Sobre el caso específico iraquí: (Cordesman \& Hashim, 1997; Ismael \& Ismael, 2005). 
De acuerdo a lo anterior, se evidencia cómo la ONU inicia la creación de sanciones más precisas en contra de los movimientos financieros de individuos que hacían parte de regímenes poco legítimos o criminales, y de sus familiares ${ }^{13}$. Bajo esta directriz, la Resolución 1267/ 1999 (CS) establece sanciones financieras concretas contra los talibanes, y se impone un bloqueo aéreo a sus aeronaves. Estos fueron acusados de poner en peligro la paz y seguridad internacionales, al brindar refugio y entrenamiento a terroristas internacionales, y además por su negativa de entregar a Osama Bin Laden, quien había sido acusado de organizar los ataques de las embajadas estadounidenses en Kenia y Tanzania en 1998, que habían dejado un saldo de 224 muertos.

Pero es la Resolución 1333/2000 (CS) la que, en palabras de Hinojosa, "desterritorializa" las sanciones del Consejo de Seguridad, porque además de reforzar las acciones en contra de los talibanes, solicita que todos los estados congelen los fondos y otros activos financieros de Osama Bin Laden y de las personas y entidades asociadas a él, incluida la organización Al Qaeda, y además pide el mantenimiento de una lista actualizada de estas personas, de acuerdo a la Resolución 1267 (CS) de 1999 (Hinojosa, 2008, p. 121).

\section{CONCLUSIONES}

No ha sido fácil consolidar un régimen internacional que controle la financiación del terrorismo internacional. La tecnología ha sido un aspecto paradójico; por una parte ha facilitado la creación de mecanismos como el GAFI, y de servicios en línea para vigilar transacciones sospechosas, pero a su vez ha facilitado que las redes terroristas operen sin dejar huella en diversas regiones del sistema internacional.

La escala de daño del terrorismo internacional ha crecido de manera vertiginosa. De la desviación y secuestro de aeronaves, y atentados tradicionales contra infraestructura específica durante la Guerra Fría, se ha pasado a voluminosos y espectaculares ataques como los ocurridos en Nueva York (11-S), Madrid (11-m) y Bali (2002). También resulta paradójico el relativo bajo costo de estas operaciones; no obstante, la ONU y algunos de sus miembros se han especializado en combatir las diversas formas legales e ilegales, que existen para financiar dichas acciones.

Esta organización continúa liderando un régimen de control de financiación del terrorismo, que se apoya también en otras iniciativas como el GAFI. Las diversas sanciones que llegan a "desterritorializarse" se hacen más específicas durante las décadas de 1980 y 1990, pero no logran detener las pretensiones de Al Qaeda en suelo estadounidense en 2001.

13. Un interesante trabajo en el que se analiza el papel de la ONU frente al terrorismo es: (Boulden, 2007). 
Una de las consecuencias del 11-S fue la creación del Comité Contra el Terrorismo ${ }^{14}$ concebido a través de la Resolución 1373 de 2001 (CS), el cual desde entonces ha condenado atentados en diversas zonas del planeta (Bali, Moscú y Kenia en 2002, Bogotá y Estambul, en 2003, Londres e Irak en 2005), lo cual demuestra la difícil naturaleza y continuidad de la problemática que este régimen intenta controlar.

Tal vez sea crítico para un sistema internacional multipolar, que parte del régimen sea concebido por las potencias tradicionales del Consejo de Seguridad. En los próximos años será vital la cooperación de otros actores emergentes y tradicionales que también sufren las consecuencias del terrorismo y su financiación, tales como: Indonesia, India, China, Pakistán, Japón, Italia y Colombia, entre otros, los cuales pueden contribuir de manera significativa para que el régimen adopte un perfil más multilateral, efectivo y anticipatorio, y lo más importante, sin que afecte los principios del derecho internacional.

\section{BIBLIOGRAFÍA}

- Boulden, J. (2007). Terrorism. In Weiss, T. G. \& Daws, S. (Eds.), The Oxford Handbook on the United Nations (pp. 427-436): Oxford University Press, USA.

- $\quad$ Bunyan, T. (1993). Trevi, Europol and the European state. Statewatching the new Europe a Handbook on the European State(13), 15.

- Buzan, B. (1993). From International System to International Society: Structural Realism and Regime Theory Meet the English School. International Organization, 47(3), 327-352.

- Byers, M. (2002). Terrorism, the use of force and international law after 11 September. International Relations, 16(2), 155.

- Cordesman, A. H., \& Hashim, A. (1997). Iraq: Sanctions and beyond. Boulder, Colorado: Westview Press.

- Curia, E. (2005). Control y Prevención del Lavado de Dinero. Retrieved 7 de mayo de 2008, from http://www.pwc.com/ar/spa/pdf/CEO_LavadoDinero.pdf

- Drezner, D. W. (1999). The Sanctions Paradox: Economic Statecraft and International Relations. Cambridge: Cambridge University Press.

14. Para más información véase su sitio en internet: http://www.un.org/es/sc/ctc/

EVOLUCIÓN DEL RÉGIMEN DE CONTROL Y FINANCIACIÓN DEL TERRORISMO 
- Haggard, S., \& Simmons, B. (1987). Theories of international regimes. International Organization, 41(03), 491-517.

- Hasenclever, A., Mayer, P., \& Rittberger, V. (1997). Theories of international regimes. Cambridge; New York: Cambridge University Press.

- Hinojosa, L. (2008). La Financiación del Terrorismo y las Naciones Unidas. Madrid: Tecnos.

- Ismael, T., \& Ismael, J. (2005). Whither Iraq? Beyond Saddam, sanctions and occupation. Third World Quarterly, 26, 609-629.

- Jervis, R. (1982). Security Regimes. International Organization, 36(2), 357-378.

- Jordan, J. (2004). Profetas del Miedo. Aproximación al terrorismo islamista. Barañáin, Navarra: Ediciones Universidad de Navarra.

- Joseph, J. (2009). Critical of what? Terrorism and its study. International Relations, 23(1), 93.

- Keohane, R. O. (1982). The Demand for International Regimes. International Organization, 36(2), 325-355.

- Keohane, R. O. (2002). The Globalization of Informal Violence, Theories of World Politics, and the "Liberalism of Fear". Dialog-IO, 29-43.

- $\quad$ Krasner, S. D. (1983). International regimes. Ithaca: Cornell University Press.

- Lopez, G. A., \& Cortright, D. (2004). Containing Iraq: Sanctions Worked. Foreign Affairs, 83(4), 90-103.

- Marsella, A. (2004). Reflections on Internacional Terrorism: Issues, Concepts and Directions. In Moghadam, F. \& Anthony, M. (Eds.), Understanding Terrorism. Washington: American Psicological Association.

- Mowlan, M. (2007). Global Terrorism. In Hocking, J. \& Lewis, C. (Eds.), Counter-Terrorism and the Post-Democratic State. UK: Edwar Elgar Publishing.

- Mueller, J., \& Mueller, K. (1999). Sanctions of Mass Destruction. Foreign Affairs, 78(3), 4353.

- Pape, R. A. (1997). Why Economic Sanctions Do Not Work. International Security, 22(2), 90-136. 
- Pape, R. A. (1998). Why Economic Sanctions Still Do Not Work. International Security, 23(1), 66-77.

- $\quad$ Reeve, S. (2000). One day in September: the full story of the 1972 Munich Olympics massacre and the Israeli revenge operation" Wrath of God": Arcade Publishing.

- Schmid, A. (1993). The Response Problem as a Definition Problem. In Schmid, A. \& Crelinsten, R. (Eds.), Western Responses to Terrorism. London: Frank Cass.

- Wight, C. (2009). Theorising terrorism: the state, structure and history. International Relations, 23 (1), 99-106. 